\title{
Radio Interoperability in 5G and 6G Multiradio Base Station
}

\author{
M. E. Leinonen, M. Jokinen, N. Tervo, O. Kursu and A. Pärssinen \\ University of Oulu, Centre for Wireless Communications \\ \{marko.e.leinonen, markku.jokinen, nuutti.tervo, olli.kursu, aarno.parssinen\}@oulu.fi
}

\begin{abstract}
The 5G system enables flexible and high data rate communication between nearby vehicles, infrastructure nodes, or pedestrians to enable smart traffic use-cases. However, fully autonomous driving vehicles supporting real-time, high-quality, and high data rate sensor data sharing will require data rates beyond 5G, which a future 6G system will support. Mobile devices (UE) and base stations (BTS) supporting the $5 \mathrm{G}$ and the future 6G are implemented as multi-mode devices, where multiple radios supporting different systems are integrated into one. The highest 5G data rates are enabled with 5G millimeter wave (mmW) radios, but those face interoperability challenges of harmonic transmissions (TXs) of Long Term Evolution (LTE) and Wi-Fi radios. The 6G radios will suffer co-channel interference from the fundamental TXs of legacy cellular systems since an analog baseband signal of $6 \mathrm{G}$ radio will be wider than the frequency allocations of current wireless systems. Similarly, the fundamental TXs of LTE and Wi-Fi may introduce co-channel interference to the analog intermediate frequency (IF) interface of $5 \mathrm{G} \mathbf{m m W}$.

A minimum $111 \mathrm{~dB}$ isolation requirement is calculated for the co-channel interference from the LTE antenna to the $5 \mathrm{G} \mathrm{mmW}$ analog IF interface. The measured isolation in the $5 \mathrm{G} \mathrm{mmW}$ proof-of-concept radio was $67 \mathrm{~dB}$, which is significantly lower than the requirement. The $5 \mathrm{G} \mathrm{mmW}$ OTA link measurements show that a narrow interference can block the $5 \mathrm{G} \mathrm{mmW}$ operation if the IF signal has co-channel interference distorting the $5 \mathrm{G}$ synchronization signals. The $5 \mathrm{G} \mathrm{mmW}$ analog interface measurement verifies that the future 6G multi-mode UEs and BTSs will face severe radio interoperability challenges with $5 \mathrm{G}$, LTE, and Wi-Fi transmissions.
\end{abstract}

Keywords - Base station. Error Vector Magnitude, Interface, Interference, Mobile device

\section{INTRODUCTION}

Fifth-generation (5G) mobile networks will offer ten-fold data rates compared with the current Long Term Evolution (LTE) system. The 5G system will offer $20 \mathrm{Gbps}$ and $10 \mathrm{Gbps}$ peak data rates for downlink (DL) and uplink, respectively. The highest data rates require wide signal bandwidths (BWs) available at millimeter-wave $(\mathrm{mmW})$ frequency bands. Current third generation partnership project (3GPP) 5G new radio (NR) standard supports signal BWs up to $400 \mathrm{MHz}$ at mmW frequencies [1], [2]. The widest supported intra-band carrier aggregation combination in the current mobile terminal standard is $1200 \mathrm{MHz}$ [2], and future $5 \mathrm{G}$ releases will enhance data rates. Wireless communication standards beyond $5 \mathrm{G}$ towards $6 \mathrm{G}$ will require significantly wider signal $\mathrm{BWs}$ to support continuously growing data rate demand. A lower $\mathrm{THz}$ frequency band between $200-300 \mathrm{GHz}$ is one of the candidate bands for future $6 \mathrm{G}$ high data rate communication. It has been envisioned that the data rates of the $6 \mathrm{G}$ system can reach up to
1 Tbps [3]. Even though no official 6G standard exists, the first proposal for the low-THz wireless standard IEEE 802.15.3d is available, where the widest carrier aggregated signal BW is $69.2 \mathrm{GHz}$ [4]. A one-meter wireless link operating at a $100 \mathrm{Gbps}$ data rate at $230 \mathrm{GHz}$ frequency with a single carrier 16-QAM modulation has been demonstrated in [5]. One of the first use-cases which have been envisioned for $6 \mathrm{G}$ relates to autonomous driving and vehicles [3]. The 5G standard has addressed the autonomous driving with a standard [6], which specifies minimum requirements for use-cases. The highest data rates are needed for an imminent collision scenario, where fully automated vehicles are sharing real-time sensor data directly between vehicles or to the network via a roadside unit. The maximum currently specified data rate, such as $5 \mathrm{G}$ use-case is $1 \mathrm{Gbps}$, but it will be enhanced, when higher data rates are widely available in the $5 \mathrm{G}$ and future $6 \mathrm{G}$ networks. Current vehicle to vehicle communication utilizes additional Wi-Fi communication path based on IEEE 802.11p with $5 \mathrm{G}$ and LTE communications to enhance overall data rate and reliability [7]

Current 5G mobile terminals (UEs) and small cell base stations (BTSs) support multiple wireless technologies simultaneously. A trend for $5 \mathrm{G} \mathrm{mmW}$ UE receivers $(\mathrm{Rx})$ is that they will not utilize filters [8], [9] in mmW RF front end. Implementation of $5 \mathrm{G} \mathrm{UE}$ with multiple $\mathrm{mmW}$ antenna arrays has been studied in [10]. Interoperability requirements between different radio systems, e.g., Wi-Fi, LTE, and 5G, has not been standardized by $3 \mathrm{GPP}$ or by any other standardization body. An interference scenario, where the interference signals are coupled from the LTE or Wi-Fi antennas to the $5 \mathrm{G} \mathrm{mmW}$ antenna, is studied in [11].

A wideband analog signal interface (i/f) is vulnerable to narrow interference signals, as shown in the example in Fig. 1. It can be seen that the $5 \mathrm{G} \mathrm{mmW}$ radio suffers from the co-channel interferences of LTE and Wi-Fi transmissions (TXs) at an intermediate frequency (IF) or from their harmonic interferences at $\mathrm{mmW}$ frequency. The $6 \mathrm{G}$ analog $\mathrm{BB}$ reception (RX) will suffer co-channel interference from fundamental frequency $\mathrm{TX}$ of $5 \mathrm{G}$, LTE, and $\mathrm{Wi}-\mathrm{Fi}$ and harmonic TXs of LTE and Wi-Fi. The analog BB signal is present in the interconnection cable, at the signal trace or inside of the IC, depending on the system partitioning and architecture.

It can be seen from Fig. 1 that radio interoperability can be implemented by optimizing the RX IF frequency of the $5 \mathrm{G} \mathrm{mmW}$ radio towards LTE interference. However, when 


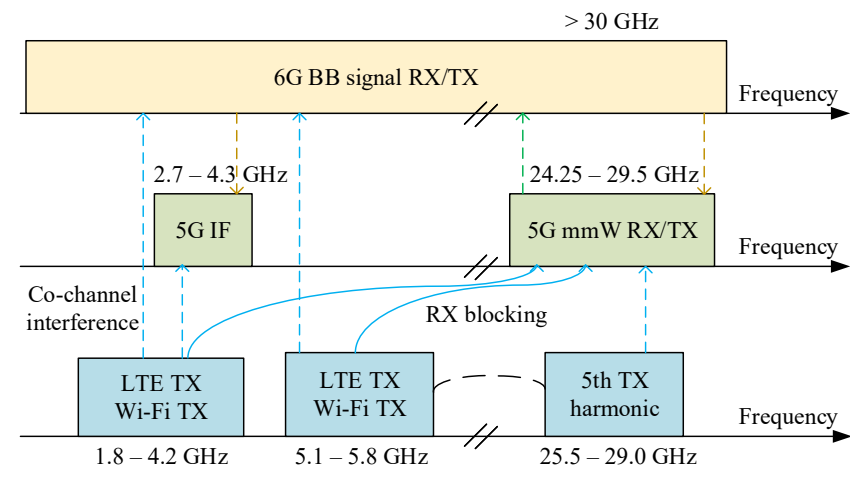

Fig. 1. 6G, 5G, LTE and Wi-Fi interference scenarios.

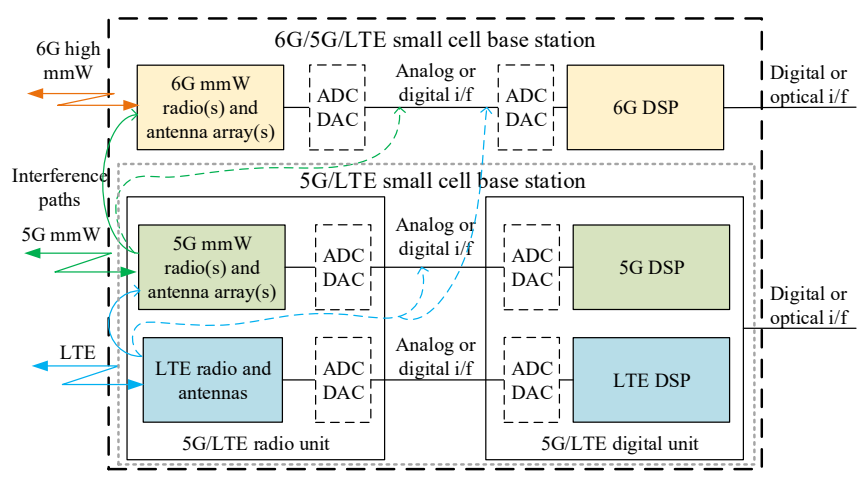

Fig. 2. Block diagram of $6 \mathrm{G}$ multi-mode base station with interference paths.

the supported BWs of $5 \mathrm{G}$ systems get wider, the frequency planning of non-overlapping frequency allocations becomes more demanding or even impossible. For this reason, the interference scenarios in the analog interconnection between LTE, Wi-Fi, 5G, and 6G need to be studied. This paper shows that more than $110 \mathrm{~dB}$ isolation is needed from the LTE transmission antenna to the $5 \mathrm{G} \mathrm{mmW}$ analog interface to enable seamless concurrent operation between $5 \mathrm{G} \mathrm{mmW}$ and LTE. The same analysis can be extended to the $6 \mathrm{G}$ radio system, as well.

\section{INTERFERENCE SCENARIO IN 6G MULTIRADIO BTS}

A block diagram of the future $6 \mathrm{G}$ multi-mode small cell BTS, which can be used, for example, as a road-side unit in the traffic use-case, is shown in Fig. 2. The 6G BTS can be divided into two main parts: $6 \mathrm{G}$ radio and $5 \mathrm{G}$ multi-mode small cell BTS, which has similar functionalities as currently deployed units. The $6 \mathrm{G}$ radio will have a dedicated digital interface to the core network since the supported data rates are significantly higher than $5 \mathrm{G}$ data rates. It can be seen from the block diagram that the LTE and 5G TXs may couple to the analog $\mathrm{i} / \mathrm{f}$ of the $6 \mathrm{G}$ radio, and similarly, the LTE or Wi-Fi TX may interfere with the analog $\mathrm{i} / \mathrm{f}$ of the $5 \mathrm{G} \mathrm{mmW}$ radio. The digital signal processing (DSP) unit may be a separate unit or integrated within the radio unit. In the former case, the analog $\mathrm{i} / \mathrm{f}$ may locate between radio and DPS units or within the radio unit depending on the locations of the analog to digital (ADCs) and digital to analog converters (DACs).

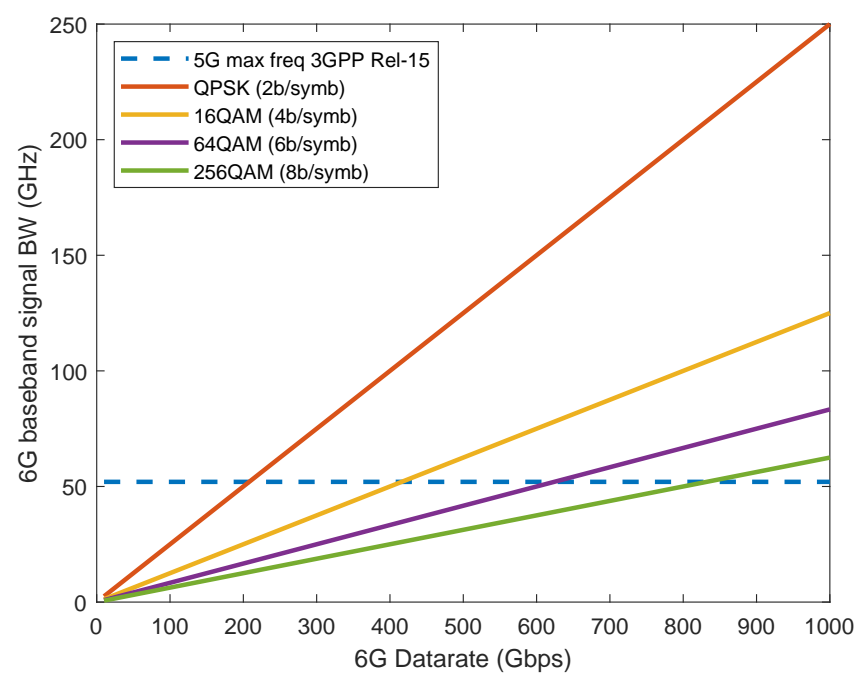

Fig. 3. Real analog $6 \mathrm{G}$ baseband signal BW with different modulations.

The signal BWs in the $6 \mathrm{G}$ analog and digital interfaces are considerably wider than in $5 \mathrm{G}$ or LTE radios. Thus, co-channel interference from other radios is easily coupled to the 6G RX signal. It can be seen from Fig. 3, that the higher the modulation order is, the more narrow analog $\mathrm{BB}$ signal BW can be used. High order modulations e.g., 64-QAM and 256-QAM, require a high signal to noise ratio for successful communication, which is difficult to achieve with currently used $200 \mathrm{GHz}$ technologies. The maximum $5 \mathrm{G}$ frequency of $52.6 \mathrm{GHz}$ from the 3GPP 5G NR standard is marked with a dotted line in Fig. 3, which indicates that 5G and LTE TXs will have significant overlap with the $6 \mathrm{G} \mathrm{BB} \mathrm{RX}$ signal with all data rates. There is no successful $6 \mathrm{G}$ communication with most of the modulations, if overlapping interference frequencies are low-pass filtered from the 6G BB signal. The real analog BB signal $\mathrm{BW}$ calculated as

$$
B W_{B B}=\frac{B W_{M o d}}{2}=\frac{B W_{\text {data }}}{2 M}
$$

where $\mathrm{BW}_{\text {data }}$ is the data rate and $\mathrm{M}$ is number of the bits per symbol, which depends on used modulation.

The interference scenarios within the $6 \mathrm{G}$ BTS can be analyzed separately for $6 \mathrm{G}, 5 \mathrm{G}$, and LTE operations. The co-channel interference scenario in the analog $\mathrm{i} / \mathrm{f}$ is analyzed in the scenario where the sub-6 GHz LTE Tx overlaps with the $5 \mathrm{G} \mathrm{mmW}$ Rx signal at IF frequency. The isolation between LTE and $5 \mathrm{G} \mathrm{mmW}$ operations in the developed $5 \mathrm{G} \mathrm{mmW}$ proof-of-concept $(\mathrm{PoC})$ radio unit has been analyzed in Table 1. It is assumed that the maximum LTE transmission level is $23 \mathrm{dBm}$ [12]. The antenna gain of the LTE antenna is assumed to be $0 \mathrm{dBi}$ for omnidirectional cell coverage. The noise figure (NF) of the $5 \mathrm{G} \mathrm{mmW}$ receiver is assumed to be $5.0 \mathrm{~dB}$ and the gain of the Rx from the antenna to the IF port of the radio $17.0 \mathrm{~dB}$, as described in [13]. Only $0.1 \mathrm{~dB}$ deterioration of the $5 \mathrm{G} \mathrm{mmW} \mathrm{RX} \mathrm{IF} \mathrm{signal} \mathrm{is} \mathrm{allowed} \mathrm{due} \mathrm{to} \mathrm{the} \mathrm{co-channel} \mathrm{LTE}$ signal interference since the interference is introduced into the 
Table 1. Calculation of maximum interference in $5 \mathrm{G} \mathrm{mmW}$ IF interface.

\begin{tabular}{|l|l|l|l|}
\hline & Variable & Value & unit \\
\hline A & $5 \mathrm{G} \mathrm{mmW} \mathrm{signal} \mathrm{BW}$ & 100 & $\mathrm{MHz}$ \\
\hline B & Thermal noise (ktB) & -174 & $\mathrm{dBm} / \mathrm{Hz}$ \\
\hline C & NF of array Rx & 5.0 & $\mathrm{~dB}$ \\
\hline D & Noise level in antenna input (A*B+C) & -89.0 & $\mathrm{dBm}$ \\
\hline E & RX gain from mmW to IF port & 17.0 & $\mathrm{~dB}$ \\
\hline F & Noise level in IF cable (D+E) & -72.0 & $\mathrm{dBm}$ \\
\hline G & Max noise raise due to interference & 0.1 & $\mathrm{~dB}$ \\
\hline H & Noise + interference level (F+G) & -71.9 & $\mathrm{dBm}$ \\
\hline I & Max interference level Eq. (2) & -88.3 & $\mathrm{dBm}$ \\
\hline $\mathrm{J}$ & Max LTE TX level with 0 dBi antenna & 23.0 & $\mathrm{dBm}$ \\
\hline K & Isolation from LTE to 5G cable (J-I) & 111.3 & $\mathrm{~dB}$ \\
\hline
\end{tabular}

back-end of the RF signal chain. The maximum interference level in the $5 \mathrm{G} \mathrm{mmW} \mathrm{i/f} \mathrm{cable} \mathrm{can} \mathrm{be} \mathrm{calculated}$

$$
I_{\max }=10 \log _{10}\left(10^{(H / 10)}-10^{(F / 10)}\right)
$$

where $\mathrm{H}$ is combined noise and interference level, as shown at row $\mathrm{H}$ in Table 1 , and the $\mathrm{F}$ is the noise level in the cable as shown at row $\mathrm{F}$ in Table 1 . It can be concluded that at least $111 \mathrm{~dB}$ isolation is needed from the LTE antenna inside of the $5 \mathrm{G} \mathrm{mmW} \mathrm{i} / \mathrm{f}$ cable. The isolation can be improved by optimizing the location of the LTE antenna, by using shielding around the $\mathrm{i} / \mathrm{f}$ coaxial cable, by selecting a high isolation coaxial cable or by using optical interconnections.

The same method, as shown in Table 1, can be applied for the $6 \mathrm{G}$ co-channel interference and isolation calculations. Ratios of the signal BWs attenuate the co-channel interference from the $6 \mathrm{G}$ BB $\mathrm{TX}$ signal to the $5 \mathrm{G} \mathrm{RX}$ signal. For example, an co-channel interference from $30 \mathrm{GHz}$ wide $6 \mathrm{G}$ signal to $100 \mathrm{MHz} 5 \mathrm{G}$ signal is attenuated by $24.8 \mathrm{~dB}$, and thus the $6 \mathrm{G}$ $\mathrm{RX}$ is the most prone for co-channel interferences.

\section{NARROW BAND SySTEM GENERATEd CO-CHANNEL INTERFERENCE TO WIDE BAND SYSTEM}

A narrow band interference scenario of a local oscillator leakage of $230 \mathrm{GHz}$ transceiver is analysed in [14], where the narrow band interference is $17 \mathrm{~dB}$ higher than average signal power of the communication signal. The $6 \mathrm{G}$ capable radio devices are not available, yet, and thus a narrow-band co-channel interference scenario and effect to a wider signal is studied with a $5 \mathrm{G} \mathrm{mmW}$ and LTE scenario, which is validated with implemented 5G mmW PoC unit [13].

Over-the-air (OTA) interference measurement between the LTE and $5 \mathrm{G} \mathrm{mmW}$ systems has been performed based on the block diagram shown in Fig. 4. The interference measurement emulates a real use-case where the $5 \mathrm{G} \mathrm{mmW}$ radio communicates with another $5 \mathrm{G} \mathrm{mmW}$ unit by using a 5G NR $100 \mathrm{MHz}$ wide Cyclic-Prefix Orthogonal Frequency Division Multiplexing (CP-OFDM) modulated signal. The interfering continuous wave $(\mathrm{CW})$ signal was transmitted, via a wideband test antenna, to emulate a narrow-band interference, such as LTE or Wi-Fi, whose BW varies from $200 \mathrm{kHz}$

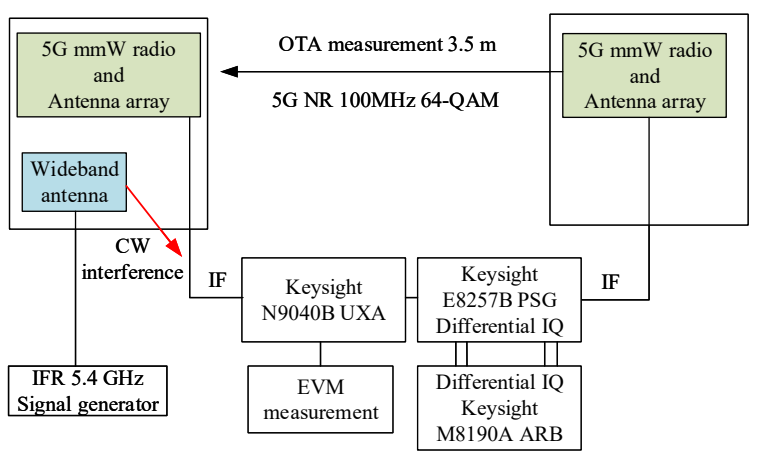

Fig. 4. 5G mmW OTA interoperability measurement setup.

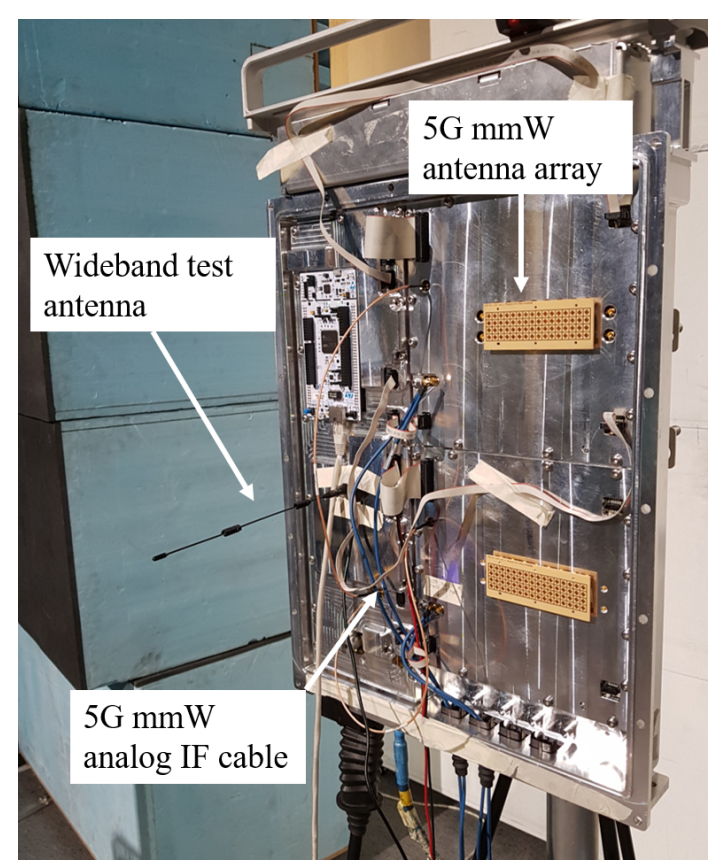

Fig. 5. Photograph of 5G and LTE interference measurement arrangement.

up to $20 \mathrm{MHz}$. The $\mathrm{CW}$ interference was used in the interference measurement since it is the worst-case interferer where all RF power is concentrated on a single frequency. The OFDM signal is composed of multiple narrow sub-carriers, which are individually modulated and demodulated. Thus, the OFDM signal should be by nature, resistant to the CW-like interference.

The photograph of the measurement arrangement is shown in Fig. 5. The interference signal antenna is located next to two $5 \mathrm{G} \mathrm{mmW}$ array $\mathrm{Rxs}$ in the radio unit. Both $5 \mathrm{G} \mathrm{mmW}$ radio Rxs have own analog $\mathrm{i} / \mathrm{f}$ connected to measurement equipment. The $5 \mathrm{G} \mathrm{mmW} \mathrm{Rx}$ operates on $4 \mathrm{GHz}$ IF frequency in all measurements. The interfering TX signal level was set to $+13 \mathrm{dBm}$.

\section{A. Interference Measurements at IF frequency of $5 G \mathrm{mmW}$}

The interfering $\mathrm{CW}$ signal was swept from $2.6 \mathrm{GHz}$ to $5.4 \mathrm{GHz}$ by $100 \mathrm{MHz}$ steps to cover high-frequency LTE bands. The coupling of interference signal to the coaxial cable 


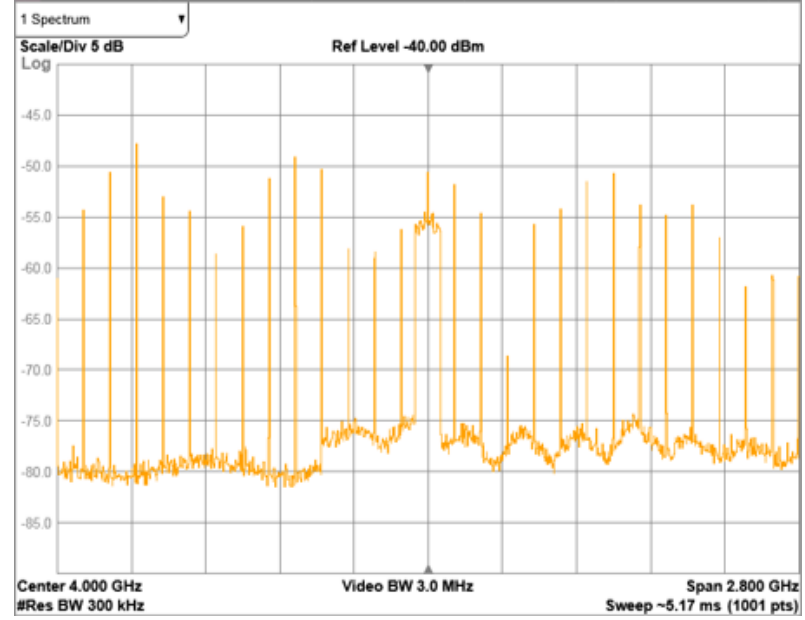

Fig. 6. OTA coupled interference signal levels in $5 \mathrm{G} \mathrm{mmW}$ analog interface coaxial cable.

was measured with a spectrum analyzer with a max hold setting. It can be seen from Fig. 6 that the interference signal is strongly coupled to the coaxial interface, since the interference signal level is $5 \mathrm{~dB}$ higher than the received $5 \mathrm{G} \mathrm{mmW}$ signal at IF frequency. The measured radiated isolation from the measurement antenna to the coaxial cable at IF frequency was $13 \mathrm{dBm}-(-50 \mathrm{dBm})=63 \mathrm{~dB}$, which is $48 \mathrm{~dB}$ lower than the calculated isolation requirement in Table 1.

The coupling mechanism was studied by placing a finger over the cable and coaxial connector. The finger placement over the coaxial connector improved the measured isolation by 7 to $15 \mathrm{~dB}$ depending on the frequency. The coaxial connectors need to be adequately shielded to improve radio interoperability.

\section{B. Measured $5 G \mathrm{mmW}$ Performance with Presence of Narrow Band Co-channel Interference}

The $5 \mathrm{G} \mathrm{mmW}$ link performance with presence of the narrow band interference at IF frequency was measured with the error vector magnitude (EVM). The EVM measurement was performed with multiple $\mathrm{CW}$ interference signal offsets from the center of the $5 \mathrm{G} \mathrm{mmW}$ IF frequency. An example EVM measurement is shown in Fig. 7, where the CW frequency is set $2.0 \mathrm{MHz}$ above the center of the used $5 \mathrm{G}$ $\mathrm{mmW}$ IF frequency.

It can be seen from Fig. 7 that even $\mathrm{CW}$ interference may ultimately stop the $5 \mathrm{G} \mathrm{mmW}$ communication, since more than 200 adjacent sub-carriers were affected by the interference. Results were similar when the frequency of the CW interferer was shifted within the BW of the $100 \mathrm{MHz}$ wide $5 \mathrm{G}$ NR signal. The CW signal can completely distort the synchronization and the pilot signals, as shown in Fig. 6 and in Table 2.

Thus, any CW interference source e.g. clock harmonics of digital processors or converters may introduce new interoperability problems with $5 \mathrm{G}$ and future $6 \mathrm{G}$ wideband communication signals. Interference signals may couple to the baseband communication signal either over-the-air

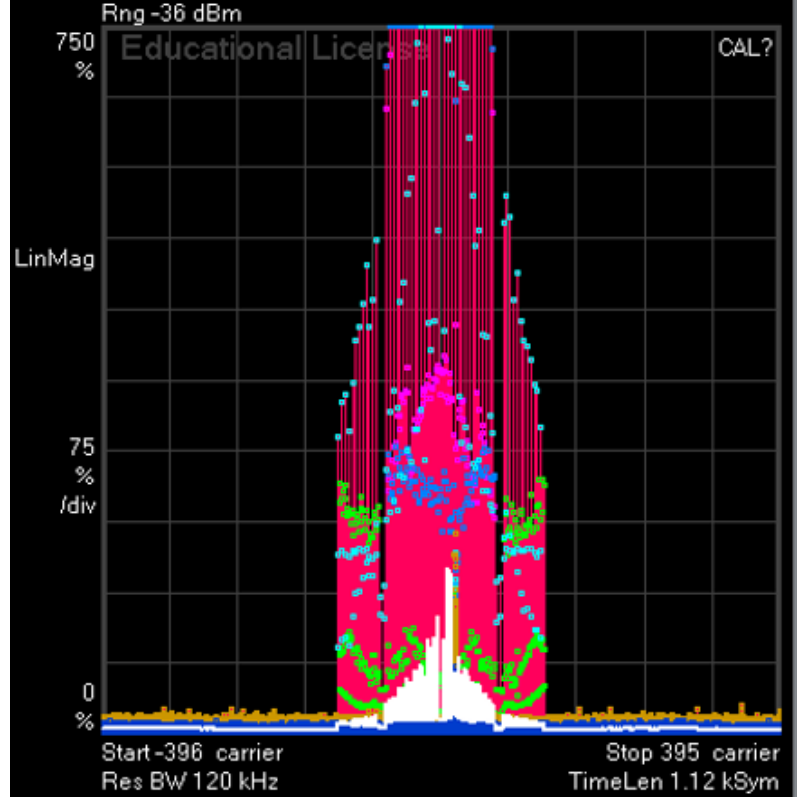

Fig. 7. Sub-carrier EVM plot of $100 \mathrm{MHz} 5 \mathrm{G} \mathrm{NR}$ signal with co-channel $\mathrm{CW}$ interference at $2.0 \mathrm{MHz}$ apart from the RF channel center frequency.

Table 2. 5G NR EVM measurements with co-channel CW interference.

\begin{tabular}{|l|l|l|l|}
\hline Channel name & $\begin{array}{l}\text { EVM } \\
(\% \text { rms })\end{array}$ & Modulation & Number \\
\hline PSS (Primary Sync. Signal) & 275.58 & BPSK & 48 \\
\hline SSS (Secondary Sync. Signal) & 321.53 & BPSK & 48 \\
\hline PBCH (Physical Broadcast Ch.) & 38.83 & QPSK & 80 \\
\hline $\begin{array}{l}\text { PBCH DMRS (Demod. Reference } \\
\text { Signal) }\end{array}$ & 166.6 & BPSK & 80 \\
\hline PDSCH (Physical DL Shared Ch.) & 5.64 & 64QAM & 520 \\
\hline PDSCH DMRS & 4.96 & QPSK & 520 \\
\hline
\end{tabular}

or conductively via ground or operational voltage planes or control signals. These topics need to be taken into consideration in the 6G RF IC and the printed board designs.

\section{CONCLUSION}

The introduction of the $5 \mathrm{G} \mathrm{mmW}$ system with LTE and Wi-Fi generates new radio interoperability problems. The fundamental frequency LTE TX creates co-channel interference at the IF frequency of the $5 \mathrm{G} \mathrm{mmW}$ radio. Future BB signal of the $6 \mathrm{G}$ system will suffer the co-channel interference from all previous 5G, LTE, and Wi-Fi systems. It was calculated that $111 \mathrm{~dB}$ isolation from the LTE TX to the $5 \mathrm{G} \mathrm{mmW}$ coaxial cable analog interface is needed to guarantee simultaneous operation. The measured isolation in the $5 \mathrm{G}$ $\mathrm{mmW}$ PoC system from the LTE antenna to $5 \mathrm{G} \mathrm{mmW}$ interface was $63 \mathrm{~dB}$ indicating that design improvements are needed. Additionally, it was demonstrated that the $\mathrm{CW}$ co-channel interference may disrupt the $5 \mathrm{G} \mathrm{mmW}$ connection by distorting the synchronization signals. 


\section{ACKNOWLEDGMENT}

This work was supported in part by the Academy of Finland 6Genesis Flagship (grant no. 318927) and in part Business Finland funded projects 5G-VIIMA and 5G-FORCE.

\section{REFERENCES}

[1] 3GPP, "NR; Base Station (BS) radio transmission and reception (Release 15)," 3GPP, Technical Specification (TS) 38.104, 12 2018, version 15.2.0.

[2] — - "NR; User Equipment (UE) radio transmission and reception Part 2: Range 2 Standalone (Release 15)," 3GPP, Technical Specification (TS) 38.101, 12 2018, version 15.2.0.

[3] M. Latva-aho and K. Leppänen, Key drivers and research challenges for 6 Gubiquitous wireless intelligence. Univ. of Oulu, Finland, 2019.

[4] IEEE, "IEEE Standard for High Data Rate Wireless Multi-Media Networks-Amendment 2: $100 \mathrm{~Gb} / \mathrm{s}$ Wireless Switched Point-to-Point Physical Layer," IEEE Std 802.15.3d-2017 (Amendment to IEEE Std 802.15.3-2016), pp. 1-55, Oct 2017.

[5] P. Rodríguez-Vazquez, J. Grzyb, B. Heinemann, and U. R. Pfeiffer, "A 16-QAM 100-Gb/s 1-m Wireless Link with an EVM of $17 \%$ at $230 \mathrm{GHz}$ in an SiGe Technology," IEEE Microwave and Wireless Components Letters, vol. 29, no. 4, pp. 297-299, April 2019.

[6] ETSI, "5G; Service requirements for enhanced V2X scenarios (3GPP TS 22.186 version 15.3.0 Release 15)," ETSI, Technical Specification (TS) 122.186, 7 2018, version 15.3.0.

[7] L. F. et. al., "V2X White Paper," NGMN, White paper, 6 2018, version 1.0 .

[8] H. Kim et al., "A 28GHz CMOS Direct Conversion Transceiver with Packaged Antenna Arrays for 5G Cellular System," in 2017 IEEE Radio Frequency Integrated Circuits Symposium (RFIC), June 2017, pp. 69-72.

[9] B.Sadhu et al., "7.2 a 28 GHz 32-Element Phased-Array Transceiver IC with Concurrent Dual Polarized Beams and 1.4 Degree Beam-Steering Resolution for 5G Communication," in IEEE ISSCC Dig. Tech. Papers, Feb 2017, pp. 128-129.

[10] Y. Huo, X. Dong, and W. Xu, "5G Cellular User Equipment: From Theory to Practical Hardware Design," IEEE Access, vol. 5, pp. 13 992-14010, 2017.

[11] M. E. Leinonen, M. Sonkki, O. Kursu, and A. Pärssinen, "5G mmW Receiver Interoperability with Wi-Fi and LTE transmissions," in Proc. EUCAP Conf., March 2019, pp. 1-5.

[12] 3GPP, "Evolved Universal Terrestrial Radio Access (E-UTRA); Base Station (BS) radio transmission and reception (Release 15)," 3GPP, Technical Specification (TS) 36.104, 12 2017, version 15.1.0.

[13] M. E. Leinonen, M. Jokinen, N. Tervo, O. Kursu, and A. Pärssinen, "System EVM Characterization and Coverage Area Estimation of 5G Directive mmW Links," IEEE Transactions on Microwave Theory and Techniques, vol. 67, no. 12, pp. 5282-5295, Dec 2019.

[14] P. Rodríguez-Vazquez, M. E. Leinonen, J. Grzyb, N. Tervo, A. Pärssinen, and U. R. Pfeiffer, "Signal-processing Challenges in Leveraging $100 \mathrm{~Gb} / \mathrm{s}$ Wireless THz," in 2020 2nd $6 G$ Wireless Summit (6G SUMMIT), March 2020, pp. 1-5. 